\title{
Rogue waves and other solutions of single and coupled Ablowitz-Ladik and nonlinear Schrödinger equations
}

\author{
A.Ankiewicz, N. Devine, M. Ünal, A. Chowdury, and N. Akhmediev \\ Optical Sciences Group, Research School of Physics and Engineering, \\ The Australian National University, Canberra ACT 0200, Australia
}

\begin{abstract}
We provide a simple technique for finding the correspondence between the solutions of AblowitzLadik and nonlinear Schrödinger equations. Even though they belong to different classes, in that one is continuous and one is discrete, there are matching solutions. This fact allows us to discern common features and obtain solutions of the continuous equation from solutions of the discrete equation. We consider several examples. We provide tables, with selected solutions, and these allow us to easily match the pairs of solutions. We show that our technique can be extended to the case of coupled Ablowitz-Ladik and nonlinear Schrödinger (i.e. Manakov) equations. We provide some new solutions.
\end{abstract}

PACS numbers: PACS numbers: 42.65.-k, 47.20.Ky, 47.25.Qv

\section{INTRODUCTION}

The nonlinear Schrödinger (NLS) and Ablowitz-Ladik (AL) $[1,2]$ equations are amongst the most studied examples in the world of integrable equations. The latter can be considered as a special form of discretisation of the former. This special form allows us to maintain the integrability of the AL equation. Integrability provides an infinite number of exact solutions for each case. As a consequence of the correspondence between the two cases, we can reveal pairs of solutions that can represent a special discretisation of continuous solutions and their discrete analogs. In principle, such a correspondence should allow us to find the solution of one equation if the solution of the other equation is known. However, this linkage is not trivial and requires knowledge of at least several examples before we can find the rules of such correspondence.

In this regard, we should mention a few previous works that relate various discrete and continuous equations [3-9]. Hirota [3] constructed a partial difference analogue of the Korteweg - de Vries equation and found its N-soliton solutions. Date etal. [4] provided a general method for generating discrete soliton equations and applied it to several physical models, including the Heisenberg ferromagnet equation [4], a single component Kadomtsev-Petviashvili equation [5], Boussinesq and sine-Gordon equations [6], as well as the KP family [7]. Hasakado [8] considered discretization of the Davey-Stewartson system, double KadomtsevPetviashvili system and Ablowitz-Ladik hierarchy in general form. Sadakane [9] showed, in terms of free fermion operators, that the AL hierarchy and the Ruijsenaars - Toda hierarchy arise from a reduction of the twocomponent Toda lattice. Most of these papers deal with generating the discrete analogue of a continuous integrable equation and do not consider any direct correspondence between the solutions. The correspondence between the solutions of discrete and continuous equations can also be quite complicated. This can be even confusing when we deal with families of solutions that involve free parameters. Formalising this correspondence and illustrating it with relatively simple examples is the aim of this paper.

Thus, this work provides simple rules and some sets of corresponding solutions. We have restricted ourselves to just the simplest examples of the periodic and localized solutions that are most frequently used in the literature. Following these rules, one can extend the results to more elaborate cases. As an example of new solutions, we provide a few corresponding pairs for coupled Manakov-type sets of NLS and AL equations.

In order to make our message clear, we start with the rules of transformation, which at first glance appear to be trivial. Nevertheless, these rules allow us to operate with highly complicated solutions. In fact, we start with simple solutions of the two equations, and turn to more complex solutions later in the work. Such a gradual increase in complexity of the solutions allows us to see the similarity of the solutions at all levels of the construction.

We start the analysis with a single Ablowitz-Ladik equation that has only one component $\psi_{n}[10]$ :

$$
\begin{aligned}
i \frac{\partial \psi_{n}^{(1)}}{\partial t} & +\frac{1}{2 h^{2}}\left(\psi_{n-1}^{(1)}+\psi_{n+1}^{(1)}-2 \psi_{n}^{(1)}\right) \\
& +\frac{1}{2}\left(\psi_{n-1}^{(1)}+\psi_{n+1}^{(1)}\right)\left|\psi_{n}^{(1)}\right|^{2}=0 .
\end{aligned}
$$

In contrast to the common form of the $\mathrm{AL}$ equation, a real coefficient, $1 /\left(2 h^{2}\right)$, is included here. This leaves the integrability of the equation intact. One way to convert the equation to a more common form is to use the transformations

$$
t \rightarrow T h^{2} \quad \text { and } \quad \psi_{n} \rightarrow \Psi_{n} / h .
$$

Then, we have

$i \frac{\partial \Psi_{n}^{(1)}}{\partial T}+\frac{1}{2}\left(\Psi_{n-1}^{(1)}+\Psi_{n+1}^{(1)}\right)\left(1+\left|\Psi_{n}^{(1)}\right|^{2}\right)-\Psi_{n}^{(1)}=0$.

On the other hand, we obtain the same form of Eq.(3) if we take the arbitrary coefficient $h$ to be $h=1$ in Eq.(1). 
Note that the normalization on $T$ differs by a factor of 2 from that in the forms given in $[1,2,11,12]$. This allows the solutions of the AL equation to line up directly with those of the traditional form of the NLS (4).

We can use inverse relations (2) to transform a general solution of AL equation (3) with $h=1$ to a solution of (1) with arbitrary $h$. This transformation allows us to link the discrete derivative of the AL equation with the continuous derivative of the NLS. The transformation seems to be simple and natural. However, it does not work without additional assumptions in cases when the solutions are families with one or several parameters. An important point is that, when doing this, we also need to transform the solution parameters. Normally, we achieve this by multiplying each independent parameter of a solution by $h$. This procedure turns out to be the key for linking the discrete and continuous solutions. To give an example, in each solution, we commonly have a phase advance term $\exp \left[i q^{2} t\right]$. Setting the parameter $q \rightarrow q h$ and having $t \rightarrow t / h^{2}$ clearly leaves this exponential term invariant. Similar conversions can be made with all other free parameters of a solution.

Eq.(1) can be considered as a special discretisation of the NLS equation (Eq.(2.1) of [13]):

$$
i \frac{\partial \psi}{\partial t}+\frac{1}{2} \psi_{x x}+|\psi|^{2} \psi=0
$$

Clearly, transforming the discrete variable $n$ into continuous one through $n=x / h$ and taking the limit $h \rightarrow 0$ reduces Eq.(1) to Eq.(4) [14]. Using this fact we can obtain NLS solutions once we have the appropriate solution of the AL equation (3). The reverse operation cannot be applied directly. Having an exact solution of the NLS does not allow us to obtain the solution of the AL equation in a straightforward way. Nevertheless, if we have a certain solution of NLS, then we can often guess the form of the AL solution with relative ease and try to transform it back to the NLS solution.

The problem is that NLS can be discretised in many ways [16]. The AL equation (3) is only one of them. However, it is important to note that the $\mathrm{AL}$ equation, like the NLS, is integrable. This means that, as with the NLS equation, it has an infinite number of exact solutions. For example, the discrete NLS

$$
i \frac{\partial \psi_{n}^{(1)}}{\partial t}+\frac{1}{2}\left(\psi_{n-1}^{(1)}+\psi_{n+1}^{(1)}-2 \psi_{n}^{(1)}\right)+\left|\psi_{n}^{(1)}\right|^{2} \psi_{n}^{(1)}=0
$$

(labelled DNLS) is also a discretisation of the NLS but its solutions cannot be obtained on a regular basis. Thus, we leave the DNLS and other forms of discretisation alone and consider only the AL of eq. (3), comparing its solutions with those of NLS.

The above considerations show that there should be a correspondence between the solutions of the two equations, NLS and AL. Some part of this correspondence has been found in previous works $[11,12,15]$. The present work gives a direct link between the two sets of solutions and provides more examples of such correspondence. Having pairs of corresponding solutions is important from both a fundamental point of view as well as for finding solutions which are known for one case and is not known for the other. At the same time, we show that the equivalence between the solutions of the two equations cannot be complete. The AL equation has periodic solutions where the period is commensurate with the discrete lattice. When this period is small, the solution of the $\mathrm{AL}$ equation cannot be directly transformed into a solution of the NLS. However, we can find the solution of the NLS that may describe the corresponding solution of the AL. These special cases will also be considered in our work. The technique can be applied, not only to a single equation, but to sets of coupled AL and NLS equations.

\section{SIMPLE EXAMPLE: TRAVELING SOLITON SOLUTION}

In finding pairs of solutions, we start with a simple example. The well-known travelling soliton solution of a single AL (1) is:

$$
\psi_{n}^{(1)}=\frac{\sinh (h p)}{h} \operatorname{sech}(n h p-v t) \exp [i(k t+b n h)],
$$

where the velocity $v$ is given by

$$
v=\frac{1}{h^{2}} \sin (h b) \sinh (h p)
$$

while the frequency $k$ is

$$
k=\frac{1}{h^{2}}[\cos (h b) \cosh (h p)-1] .
$$

Here, independent real parameters $b$ and $p$ are arbitrary.

Taking $x=n h$ and $h \rightarrow 0$ in Eq.(6), we get $v=b p$ and $k=\frac{1}{2}\left(p^{2}-b^{2}\right)$. Thus, we obtain

$$
\begin{aligned}
\psi_{n}^{(1)}= & p \operatorname{sech}[p(x-b t)] \\
& \times \exp \left[i\left(b x+\frac{1}{2}\left(p^{2}-b^{2}\right) t\right)\right],
\end{aligned}
$$

which is the basic result for an NLS soliton of amplitude $p$ moving with velocity $b$.

When the velocity is zero $(b=0)$, the solution (6) reduces to

$$
\psi_{n}^{(1)}=\frac{\sinh (h p)}{h} \operatorname{sech}(h n p) \exp \left[\frac{2 i t}{h^{2}} \sinh ^{2}\left(\frac{h p}{2}\right)\right] .
$$

Taking $x=n h$ and then the limit as $h \rightarrow 0$ reduces Eq.(8) to

$$
\psi=p \operatorname{sech}(x p) \exp \left(i p^{2} \frac{t}{2}\right)
$$

which is also a solution of the NLS, Eq.(4). This simplest example demonstrates the technique of finding the pairs of solutions and serves as proof of the principle. 


\section{ENERGY CONSIDERATIONS}

For localized solutions with zero background when $n \rightarrow$ $\pm \infty$, there is an "energy invariant" that is conserved. If the background is not a zero but has a finite value $q$, we can re-define energy, since, with the usual definition, it would become infinite. It is also conserved. Namely, for a solution $\psi_{n}$, located on a zero background field, $q=0$, we define [17]:

$$
Q=\sum_{n=-\infty}^{\infty} Q_{n}
$$

where

$$
Q_{n}=\frac{h}{2}\left(\psi_{n} \psi_{n+1}^{*}+\psi_{n}^{*} \psi_{n+1}\right)
$$

Here, $q=0$ and

$$
Q_{n}=\frac{1}{h} \sinh ^{2}(h p) \operatorname{sech}(n h p) \operatorname{sech}[(n+1) h p]
$$

and

$$
Q=\frac{2}{h} \sinh (h p) .
$$

For the NLS soliton, the energy, $Q$, is the $h \rightarrow 0$ limit of this, viz. $Q=2 p$. It agrees with the value found by integrating $|\psi|^{2}$ from Eq.(9) over all $x$.

\section{SPECIAL CASES: STRICTLY PERIODIC SOLUTIONS IN TERMS OF SINE OR COSINE FUNCTIONS}

The AL equation has "periodic" solutions with periods that are incommensurate with the lattice period. Generally, the period in these solutions is a continuous variable that may coincide with the period of the lattice. In this case, the solution becomes strictly periodic. In this section, we present three special results for strictly periodic solutions of the AL equation which can be expressed in terms of sine or cosine functions.

Case (a):

$$
\psi_{n}^{(1)}=c \sin \left(\frac{n \pi}{2}\right) \exp \left(-i t / h^{2}\right),
$$

which can be presented as the pattern

$$
=[0,1,0,-1,0,1,0,-1, \cdots] \times c \exp \left(-i t / h^{2}\right)
$$

with the lattice period 4 .

Case (b). Another strictly periodic solution

$$
\psi_{n}^{(1)}=c \sin \left(\frac{n \pi}{3}\right) \exp \left[i\left(\frac{3 c^{2}}{8}-\frac{1}{2 h^{2}}\right) t\right],
$$

has the pattern

$$
\begin{aligned}
& {[0,1,1,0,-1,-1,0,1,1, \cdots] \times} \\
& \frac{\sqrt{3}}{2} c \exp \left[i\left(\frac{3 c^{2}}{8}-\frac{1}{2 h^{2}}\right) t\right]
\end{aligned}
$$

with the lattice having period 6 .

Case (c). Finally, the third strictly periodic solution

$$
\psi_{n}^{(1)}=c \sin \left(\frac{2 n \pi}{3}\right) \exp \left[-\frac{3 i}{8}\left(c^{2}+\frac{4}{h^{2}}\right) t\right],
$$

has the pattern

$$
\begin{aligned}
& {[1,-1,0,1,-1,0,1,-1, \cdots] \times } \\
& \frac{\sqrt{3}}{2} c \exp \left[-\frac{3 i}{8}\left(c^{2}+\frac{4}{h^{2}}\right) t\right]
\end{aligned}
$$

with the lattice period being 3. An arbitrary (positive real) parameter $c$ simultaneously defines the amplitude and the propagation constant of the solutions.

The strictly periodic solutions presented in this section do not have limits at $h \rightarrow 0$ because the phase variation in $\mathrm{t}$ would be infinitely rapid. In turn, the NLS does not have simple sinusoidal solutions with finite amplitude. The above expressions exist only in the discrete limit. When talking about similar solutions of the two equations, we can only consider those AL solutions with variations along $n$ much bigger than the mesh-size of the grid, $h$.

\section{PERIODIC SOLUTIONS IN TERMS OF ELLIPTIC JACOBI FUNCTIONS}

More general periodic stationary solutions can be written in terms of Jacobi elliptic functions. The variable period in these solutions may or may not coincide with the lattice period. We give three example solutions in this category [see [16] for example] with Jacobi elliptic functions with modulus $m$. The first one is in terms of the $d n$-function:

$$
\psi_{n}^{(1)}=\sqrt{a} \operatorname{dn}(b n h, m) \exp (i k t)
$$

where $m$ and $b$ are arbitrary and

$$
a=\frac{1-2 \operatorname{dn}^{2}(b h, m)+\operatorname{dn}(2 b h, m)}{h^{2} \operatorname{dn}^{2}(b h, m)[1-\operatorname{dn}(2 b h, m)]}
$$

while

$$
k=h^{-2}\left[\left(1+a h^{2}\right) \operatorname{dn}(b h, m)-1\right] .
$$

We stress that, although the elliptic Jacobi functions are periodic, the solution can be considered as periodic only when the period of the Jacobi function is commensurate with the period of the lattice. Otherwise, the periodicity is lost. When we take the limit $m=1$, we obtain the soliton solution (8) above.

To obtain the NLS solution from (16), we take the limits of $a$ and $k$ from Eqs. (17) and (18), as $h \rightarrow 0$. This transformation gives $a=b^{2}$ and $k=\left(1-\frac{m}{2}\right) b^{2}$. This provides the correct NLS solution, viz.

$$
\psi^{(1)}(x, t)=b \operatorname{dn}(b x, m) \exp \left[i\left(1-\frac{m}{2}\right) b^{2} t\right],
$$


where $m$ and $b$ are arbitrary.

A second type of periodic solution can be expressed in terms of the sn-function:

$$
\psi_{n}^{(1)}=\sqrt{a} \operatorname{sn}(b n h, m) \exp (i k t)
$$

where $m$ and $b$ are arbitrary and

$$
a=\frac{1}{h^{2} \operatorname{sn}(b, m) \operatorname{sn}(3 b, m)}-\frac{1+\frac{\operatorname{sn}(b h, m)}{\operatorname{sn}(b h, m)}}{h^{2} \operatorname{sn}^{2}(b h, m)}
$$

while

$$
k=\frac{a}{2} \operatorname{sn}(b h, m) \operatorname{sn}(2 b h, m)-\frac{1}{h^{2}}+\frac{\operatorname{sn}(2 b h, m)}{2 h^{2} \operatorname{sn}(b h, m)} .
$$

Here we need $a>0$ but

$$
\lim _{m \rightarrow 1} a<0,
$$

so this limit does not give any valid solution. The limit $h \rightarrow 0$ just gives the trivial zero solution of the NLS.

Finally, what can be called "cnoidal wave" is the following:

$$
\psi_{n}^{(1)}=\sqrt{a} \operatorname{cn}(b n h, m) \exp (i k t)
$$

where $m, b$ are arbitrary and

$$
a=\frac{1-2 \mathrm{cn}^{2}(b h, m)+\operatorname{cn}(2 b h, m)}{h^{2} \operatorname{cn}^{2}(b h, m)[1-\operatorname{cn}(2 b h, m)]}
$$

while

$$
k=h^{-2}\left[\left(1+a h^{2}\right) \operatorname{cn}(b h, m)-1\right] .
$$

When we take the limit $m=1$, we again get, as the limiting case, Eq.(8) above.

To get the NLS solution from this, we take the limits of $a$ and $k$ from Eqs. $(23,24)$, as $h \rightarrow 0$. This gives $a=m b^{2}$ and $k=\left(m-\frac{1}{2}\right) b^{2}$ and provides the correct NLS solution, viz.

$$
\psi^{(1)}(x, t)=b \sqrt{m} \operatorname{cn}(b x, m) \exp \left[i\left(m-\frac{1}{2}\right) b^{2} t\right],
$$

where $(m, b)$ are arbitrary.

In general, the period of elliptic Jacobi functions is not commensurate with the period of the lattice. However, it can be chosen to be commensurate. In this case we can obtain the strictly periodic solutions presented in the section IV. For example, let us consider the 'cn' example given by Eqs.(23) and (24). The period of the solution is $T=\frac{4}{b h} K(m)$, where $K(m)$ is the complete elliptic integral of the first kind. Now $K(m)>\frac{\pi}{2}$ so $T>\frac{2 \pi}{b h}$. If we choose $b h=K(m)$, then the period is $T=4$. Then the parameters are $k=-1 / h^{2}$ and $a=\frac{m}{h^{2}(1-m)}$. The amplitude at $t=0$ in this case can be presented as the following sequence:

$$
\psi_{n}(0)=\frac{1}{h} \sqrt{\frac{m}{1-m}} \times[0,-1,0,1,0,-1,0,1 . \cdots] .
$$

This solution can also be written in the form

$$
\psi_{n}(t)=\frac{1}{h} \sqrt{\frac{m}{1-m}} \cos \left(\frac{n \pi}{2}\right) e^{-i t / h^{2}} .
$$

which is just a shifted along $n$ solution (14) given in Section IV(a) above. Here the amplitude changes with $h$ and $m$ and can be arbitrary, just as in (14). The sequence $\psi_{n}(0)$, calculated for $h=1$ and $b=3$, is shown in Fig.1. It is labelled as $f_{4}$.

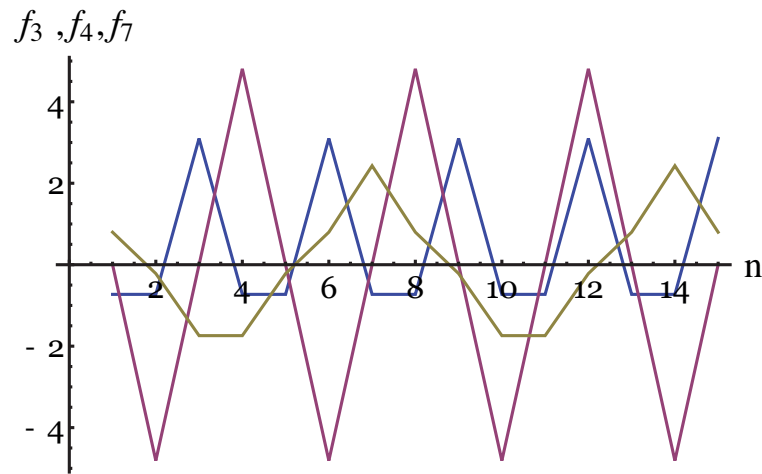

FIG. 1: Plot of the strictly periodic sequences $f_{3}$ (blue), $f_{4}$ (mauve), and $f_{7}$ (olive green) with periods 3,4 and 7 , respectively.

If we take $h=1$ and $b=4$, and choose $\mathrm{m}$ to get $T=3$, we obtain

$$
\psi_{n}=f_{3}=0.72 \times[-1,-1,4.255,-1,-1,4.255, \cdots] .
$$

We label this expression as $f_{3}$. Finally, $b=12 / 7$ gives $T=7$ and a sequence labelled $f_{7}$. All solutions are plotted in Fig.1. If we take $b h$ slightly above $2 \pi / 3$, and set $T=3$, we get $m \approx 0$ so that

$$
\operatorname{cn}(b n, m) \rightarrow \cos (2 \pi n / 3) .
$$

This way, we obtain the solution in the form presented in Section IV(c) which is shifted in $n$.

\section{MODULATION INSTABILITY AND HETEROCLINIC BREATHER SOLUTIONS}

It is well-known that the NLS has a plane-wave solution that is modulationally unstable. The family of exact solutions of the NLS that includes both the growth and decay cycles of modulation instability is known as the 'Akhmediev breathers' [24, 25, 28]. Equivalent heteroclinic solutions of the AL equation (3) have been presented and analyzed in detail in [11]. The whole family of solutions has a free parameter that corresponds to the arbitrary frequency of modulation. A particular solution of this family is related to the case when the growth rate of instability takes its maximum value. First, let us consider the latter case. 
To find the heteroclinic solution of Eq.(1) for the complete growth-return cycle of modulation instability at the maximum growth rate, we modify Eq.(15) of [12] to include the parameter $h$. Then, we will have:

$$
\begin{aligned}
& \psi_{n}(t)= \\
& q \frac{\mp \sqrt{1+h^{2} q^{2}} \cos (v n h)+i \sqrt{2+h^{2} q^{2}} \sinh \left(q^{2} t\right)}{\sqrt{2+h^{2} q^{2}} \cosh \left(q^{2} t\right) \pm \sqrt{1+h^{2} q^{2}} \cos (v n h)} e^{i q^{2} t}
\end{aligned}
$$

where

$$
v=\frac{1}{h} \arccos \left[\frac{1}{1+h^{2} q^{2}}\right]=\frac{1}{h} \operatorname{arcsec}\left[1+h^{2} q^{2}\right] .
$$

This solution describes the growth of a periodic perturbation, due to the modulation instability of the constant background, and its return to the background with a shifted phase. A qualitatively similar solution of the NLS has been derived in $[23,29]$. To obtain it from the AL solution (26), we set $n h \rightarrow x$ and then take $h \rightarrow 0$. We have

$$
\lim _{h \rightarrow 0} v=q \sqrt{2}
$$

so that:

$$
\psi(x, t)=q \frac{\mp \cos (\sqrt{2} q x)+i \sqrt{2} \sinh \left(q^{2} t\right)}{\sqrt{2} \cosh \left(q^{2} t\right) \pm \cos (\sqrt{2} q x)} e^{i q^{2} t}
$$

The above solution is a particular case of a heteroclinic orbit with an arbitrary modulation frequency, $\kappa$. The latter parameter is assumed to be within the modulation instability band. This generalization provides us with:

$$
\begin{aligned}
& \psi_{n}(t)=\frac{1}{h} \exp \left(i q^{2} t\right) \times \\
& {\left[\frac{2\left(1+h^{2} q^{2}\right) w \cosh \left(\frac{\delta t}{2 h^{2}}\right)+i \frac{\delta}{2} \sinh \left(\frac{\delta t}{2 h^{2}}\right)}{q h \cosh \left(\frac{\delta t}{2 h^{2}}\right) \pm c_{1} \cos (\kappa q n h)}-q h\right]}
\end{aligned}
$$

where

$$
w=\sin ^{2}\left(\frac{1}{2} h q \kappa\right)
$$

while

$$
c_{1}=\sqrt{\frac{q^{2} h^{2}(1-w)-w}{1-w}}
$$

and the growth rate of instability, $\delta$, is

$$
\delta=4 \sqrt{\left(1+h^{2} q^{2}\right) w\left[\left(h^{2} q^{2}(1-w)-w\right]\right.} .
$$

Clearly, Eq.(28) generalizes the solution with $h=1$ found earlier in [11].

To derive the NLS solution from (28), we take $n h=x$ and note that the limits of the coefficients are:

$$
\lim _{h \rightarrow 0}\left(\frac{w}{h^{2}}\right)=\left(\frac{q \kappa}{2}\right)^{2},
$$

$$
\lim _{h \rightarrow 0}\left(\frac{c_{1}}{h}\right)=\frac{q}{2} \sqrt{4-\kappa^{2}}
$$

and

$$
\lim _{h \rightarrow 0}\left(\frac{\delta}{h^{2}}\right)=\kappa q^{2} \sqrt{4-\kappa^{2}} .
$$

These limits allow us to write the generalization of Eq.(27) for an arbitrary modulation frequency, $\kappa$, in the range $0<\kappa<2$, as the $h \rightarrow 0$ limit of eq.(28):

$$
\begin{aligned}
& \psi(x, t)= \\
& q\left[\kappa \frac{\kappa^{2} \cosh \left(\delta_{1} q^{2} t\right)+2 i \delta_{1} \sinh \left(\delta_{1} q^{2} t\right)}{2 \kappa \cosh \left(\delta_{1} q^{2} t\right) \pm 2 \delta_{1} \cos (\kappa q x)}-1\right] e^{i q^{2} t}
\end{aligned}
$$

where the instability growth rate $\delta_{1}$ is:

$$
\delta_{1}=\frac{\kappa}{2} \sqrt{4-\kappa^{2}}
$$

in standard form. This solution satisfies the NLS for any frequency $\kappa$ in the instability range. It was first derived in $[20,29]$. In the particular case $\kappa=\sqrt{2}$, where the growth rate (30) is maximal, the expression (29) coincides with Eq.(27) above.

\section{SOLITON ON BACKGROUND}

Another important solution of the NLSE is a soliton on a background. It has been found by Kuznetsov [32] and $\mathrm{Ma}[30]$. There is a similar solution of the AL equation [12]. In order to include the parameter $h$ in the solution, we allow $k_{2}$ to have an arbitrary value and define

$$
u=\sinh \left(h k_{2} / 2\right)
$$

for convenience, so that $k_{2}=\frac{2}{h} \operatorname{arcsinh}(u)$. Generalizing the solution given in [12], we find:

$$
\begin{aligned}
& \psi_{n}(t)=\frac{1}{h} \exp \left(i q^{2} t\right) \times \\
& {\left[\frac{\cos \left(\frac{w_{1} t}{2 h^{2}}\right)+i s_{6} \sin \left(\frac{w_{1} t}{2 h^{2}}\right)}{s_{3}\left[-\cos \left(\frac{w_{1} t}{2 h^{2}}\right) \pm s_{5} \cosh \left(k_{2} n h\right)\right]}-q h\right]}
\end{aligned}
$$

where

$$
s_{3}=\frac{q h}{2\left(q^{2} h^{2}+1\right) u^{2}}
$$

and

$$
s_{5}=\sqrt{\frac{q^{2} h^{2}+\left(1+q^{2} h^{2}\right) u^{2}}{q^{2} h^{2}\left(1+u^{2}\right)}},
$$

while

$$
s_{6}=\sqrt{1+\frac{q^{2} h^{2}}{\left(q^{2} h^{2}+1\right) u^{2}}}
$$


and

$$
w_{1}=4\left(q^{2} h^{2}+1\right) u \sqrt{u^{2}+\frac{q^{2} h^{2}}{q^{2} h^{2}+1}} .
$$

Thus, in this formulation, $q, h$ and $k_{2}$ are the arbitrary real parameters. Once they are given, we can then find $s_{3}, s_{5}, s_{6}$ and $w_{1}$.

In order to transform it to the equivalent NLS solution, we first give the limiting values of the coefficients:

$$
\begin{gathered}
\lim _{h \rightarrow 0}\left(\frac{u}{h}\right)=\frac{k_{2}}{2}, \\
\lim _{h \rightarrow 0}\left(s_{3} h\right)=\frac{2 q}{k_{2}^{2}}, \\
\lim _{h \rightarrow 0} s_{5}=\frac{1}{2} \sqrt{4+\frac{k_{2}^{2}}{q^{2}}}, \\
\lim _{h \rightarrow 0} s_{6}=\sqrt{1+\frac{4 q^{2}}{k_{2}^{2}}},
\end{gathered}
$$

and

$$
\lim _{h \rightarrow 0}\left(\frac{w_{1}}{h^{2}}\right)=k_{2} \sqrt{k_{2}^{2}+4 q^{2}}
$$

Substituting these values into (31), we obtain the solution of the continuous equation which is also a soliton on a background $q$ :

$$
\begin{aligned}
& \psi(x, t)= \\
& e^{i q^{2} t}\left[k_{2} \frac{k_{2} \cos \left(\frac{k_{2}}{2} d t\right)+i d \sin \left(\frac{k_{2}}{2} d t\right)}{ \pm d \cosh \left(k_{2} x\right)-2 q \cos \left(\frac{k_{2}}{2} d t\right)}-q\right],
\end{aligned}
$$

where $d=\sqrt{k_{2}^{2}+4 q^{2}}$.

\section{ROGUE WAVES}

\section{A. First order rogue wave}

Rogue waves are doubly localized, rational solutions of the AL and NLS equations. There is an infinite hierarchy of these solutions with increasing order. For the NLS, the first-order solution has been presented by Peregrine [26] while the second order solution has been given in [29] and [18]. Rogue wave solutions of the AL equation have been presented in [11] and [12].

We consider the rogue wave solution of the AL, Eq.(1), modified from the form presented in [11]. We define $N(n)=2 q h n$ and $T(t)=2 q^{2} \sqrt{1+q^{2} h^{2}} t$. As explained in the Introduction, the two $h^{2}$ terms in $T(t)$ cancel each other. The $j$-th order rogue wave solution in general form can be written as:

$\psi_{n}^{(j)}(t)=q\left[\frac{T^{2}}{q^{4} t^{2}} \frac{G_{n}^{(j)}(t)+i 2 q^{2} t H_{n}^{(j)}(t)}{D_{n}^{(j)}(t)}+(-1)^{j}\right] e^{i q^{2} t}$

where $G_{n}^{(j)}(t)$ etc., are polynomials. For $j=1$, we have $G_{n}^{(1)}(t)=1, H_{n}^{(1)}(t)=1$ and $D_{n}^{(1)}(t)=1+N^{2}+T^{2}$. In other words:

$$
\psi_{n}^{(1)}(t)=q\left(\frac{4\left(1+h^{2} q^{2}\right)\left(1+2 i q^{2} t\right)}{1+4 h^{2} q^{2}\left(n^{2}+q^{4} t^{2}\right)+4 q^{4} t^{2}}-1\right) e^{i q^{2} t}
$$

We can use this expression to obtain the NLS rogue wave solution by taking $x=n h$, i.e. $N(n)=2 q h n=2 q x=$ $X$, and then take $h$ to be small:

$$
\psi(x, t)=q\left(\frac{4\left(1+2 i q^{2} t\right)}{1+4 q^{2} x^{2}+4 q^{4} t^{2}}-1\right) e^{i q^{2} t} .
$$

The parameter $q$ can be any real number in Eq.(35). This first order rogue wave is usually called "Peregrine soliton" $[19,25,27]$, as it was first obtained by Peregrine [26] as a limiting case of the soliton on a background (32).

\section{B. Second-order rogue wave}

Modifying the second-order rogue wave solution presented in [17] for the case of Eq.(1) that involves arbitrary $h$, we find a one-parameter family with arbitrary background level, $q$, which has the form similar to Eq.(33):

$$
\psi_{n}^{(2)}(t)=q\left[\frac{T^{2}}{q^{4} t^{2}} \frac{G_{n}^{(2)}(t)+i 2 q^{2} t H_{n}^{(2)}(t)}{D_{n}^{(2)}(t)}+1\right] e^{i q^{2} t}
$$

where

$$
\begin{aligned}
G_{n}^{(2)}(t) & =3\left[3-6\left(N^{2}-2 q^{2} h^{2}\right)\right. \\
& +N^{2}\left(4 q^{2} h^{2}-N^{2}\right) \\
& \left.-6\left(3+2 q^{2} h^{2}+N^{2}\right) T^{2}-5 T^{4}\right], \\
H_{n}^{(2)}(t) & =3\left[15+6\left(N^{2}+4 q^{2} h^{2}\right)\right. \\
& +N^{2}\left(16 q^{2} h^{2}-N^{2}\right) \\
& \left.-2\left(1+N^{2}\right) T^{2}-T^{4}\right],
\end{aligned}
$$

while

$$
\begin{aligned}
D_{n}^{(2)}(t) & =9+\left(27+24 q^{2} h^{2}+16 q^{4} h^{4}\right) N^{2} \\
& +N^{6}+3\left(33+72 q^{2} h^{2}-6 N^{2}\right. \\
& \left.+48 q^{4} h^{4}-16 q^{2} h^{2} N^{2}+N^{4}\right) T^{2} \\
& +3\left(9+8 q^{2} h^{2}+N^{2}\right) T^{4} \\
& +T^{6}+N^{4}\left(3-8 q^{2} h^{2}\right) .
\end{aligned}
$$

In order to link this solution to the second-order rogue wave of the NLS, in accordance with our technique, we 
set $n h=x$ and take $h$ to be small. Thus, as above, we denote $X=2 q x$ and $T(t)=2 q^{2} t$. We have

$$
\psi^{(2)}(x, t)=q\left[4 \frac{G^{(2)}(x, t)+i T H^{(2)}(x, t)}{D^{(2)}(x, t)}+1\right] e^{i T / 2}
$$

where

$$
\begin{aligned}
G^{(2)}(x, t) & = \\
& 3\left[3-6 X^{2}-X^{4}-6\left(3+X^{2}\right) T^{2}-5 T^{4}\right], \\
H^{(2)}(x, t) & = \\
& 3\left[15+6 X^{2}-X^{4}-2\left(1+X^{2}\right) T^{2}-T^{4}\right],
\end{aligned}
$$

while

$$
\begin{aligned}
D^{(2)}(x, t) & =9+27 X^{2}+3 X^{4}+X^{6}+T^{6} \\
& +3\left(33-6 X^{2}+X^{4}\right) T^{2}+3\left(9+X^{2}\right) T^{4} .
\end{aligned}
$$

This satisfies the NLSE and agrees with the solution in [19] and [31].

\section{COUPLED A-L AND NLS (MANAKOV) EQUATIONS}

The technique of finding equivalent solutions that we suggest in this work can be readily extended to the case of coupled AL and NLS equations. In order to demonstrate this, we write the set of two AL equations in the form:

$$
\begin{aligned}
& i \frac{\partial \psi_{n}^{(1)}}{\partial t}+\frac{1}{2 h^{2}}\left(\psi_{n-1}^{(1)}+\psi_{n+1}^{(1)}-2 \psi_{n}^{(1)}\right) \\
&+\frac{1}{2}\left(\psi_{n-1}^{(1)}+\psi_{n+1}^{(1)}\right)\left(\left|\psi_{n}^{(1)}\right|^{2}+\left|\psi_{n}^{(2)}\right|^{2}\right)=0 . \\
& i \frac{\partial \psi_{n}^{(2)}}{\partial t}+\frac{1}{2 h^{2}}\left(\psi_{n-1}^{(2)}+\psi_{n+1}^{(2)}-2 \psi_{n}^{(2)}\right) \\
&+\frac{1}{2}\left(\psi_{n-1}^{(2)}+\psi_{n+1}^{(2)}\right)\left(\left|\psi_{n}^{(1)}\right|^{2}+\left|\psi_{n}^{(2)}\right|^{2}\right)=0,
\end{aligned}
$$

where, just as before, $t$ is the continuous evolution variable (time or longitudinal spatial variable) and $n=$ $0, \pm 1, \pm 2, \cdots$ are integers labelling each cite. This set is an analog of the Manakov equations in the continuous NLS limit:

$$
\begin{aligned}
& i \frac{\partial \psi^{(1)}}{\partial t}+\frac{1}{2} \psi_{x x}^{(1)}+\left(\left|\psi^{(1)}\right|^{2}+\left|\psi^{(2)}\right|^{2}\right) \psi^{(1)}=0 \\
& i \frac{\partial \psi^{(2)}}{\partial t}+\frac{1}{2} \psi_{x x}^{(2)}+\left(\left|\psi^{(1)}\right|^{2}+\left|\psi^{(2)}\right|^{2}\right) \psi^{(2)}=0
\end{aligned}
$$

\section{STRICTLY PERIODIC SOLUTIONS}

For the set of the two AL equations, we can also write down a number of strictly periodic solutions. We can classify three of them. Namely,
Case (a): The solution with period 6 . It is given by:

$$
\psi_{n}^{(1)}=r \exp \left[i\left(\frac{3}{h^{2}}+4 r^{2}\right) t\right]\left(3-2 \cos \left(\frac{2 n \pi}{3}\right)\right),
$$

$$
\begin{gathered}
\psi_{n}^{(2)}=\frac{2}{\sqrt{5}} \sqrt{\frac{9}{h^{2}}-16 r^{2}} \exp \left[\frac{i t}{5 h^{2}}\left(11+16 h^{2} r^{2}\right)\right] \\
\times \sin \left(\frac{n \pi}{3}\right),
\end{gathered}
$$

for arbitary $r$ in the range $0<r<\frac{3}{4 h}$. Thus, we have

$\psi_{n}^{(1)}=r \exp \left[i\left(\frac{3}{h^{2}}+4 r^{2}\right) t\right] f_{1}(n)$,

$\psi_{n}^{(2)}=\sqrt{\frac{3}{5}} \sqrt{\frac{9}{h^{2}}-16 r^{2}} \exp \left[\frac{i t}{5 h^{2}}\left(11+16 h^{2} r^{2}\right)\right] \quad f_{2}(n)$,

where the sequences $f_{1}(n)$ and $f_{2}(n)$ are

$$
\begin{gathered}
f_{1}(n)=[4,4,1,4,4,1, \cdots] \\
f_{2}(n)=[1,1,0,-1-1,0,1,1, \cdots] .
\end{gathered}
$$

These sequences are strictly periodic, but the period of the second sequence is twice the period of the first one. The overall period is then 6. They are shown in Fig.2. Again, there is no physical limit for $h \rightarrow 0$, and so no corresponding NLS solution pair.

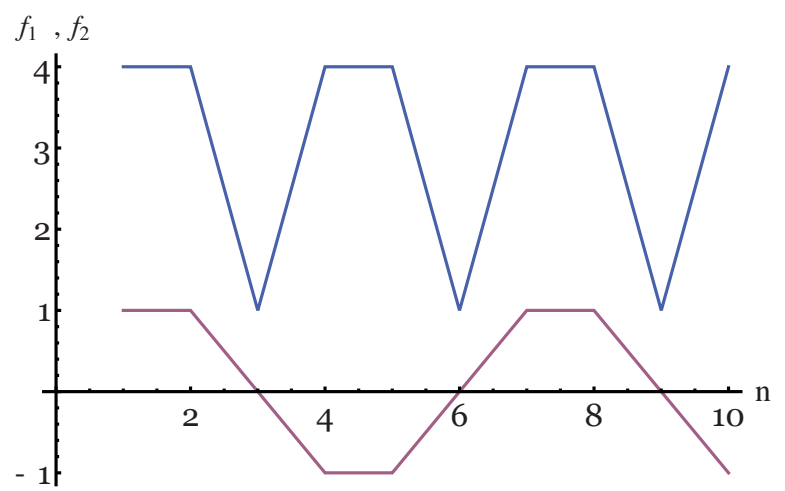

FIG. 2: Plot of $f_{1}$ (upper curve) and $f_{2}$ (lower curve) for the pair of solutions in case (a). Period is 6 .

Case (b): The solution with the period 4 . The second family is given by:

$$
\begin{aligned}
& \psi_{n}^{(1)}=d \exp (i b t)\left(3-2 g(-1)^{n}\right), \\
& \psi_{n}^{(2)}=\frac{2 \sqrt{6 g}}{h(3-2 g)} \exp \left(\frac{-i t}{h^{2}}\right) \sin \left(\frac{n \pi}{2}\right),
\end{aligned}
$$

where $b=\frac{4 g}{(3-2 g) h^{2}}+d^{2}\left(9-4 g^{2}\right)$, for arbitrary parameters $d$ and $g$. The second component (which has period 4) 
does not contain $d$ because $\psi_{n-1}^{(2)}+\psi_{n+1}^{(2)}$ is always zero. So the pattern is:

$$
\begin{aligned}
& \psi_{n}^{(1)}=d e^{i b t} \times f_{1}(n), \\
& \psi_{n}^{(2)}=\frac{2}{h(3-2 g)} \sqrt{6 g} e^{-i t / h^{2}} \times f_{2}(n)
\end{aligned}
$$

Here, we have two sequences

$$
\begin{aligned}
& f_{1}(n)=[3+2 g, 3-2 g, 3+2 g, 3-2 g, 3+2 g, \cdots] \\
& f_{2}(n)=[1,0,-1,0,1,0,-1, \cdots]
\end{aligned}
$$

with the period of the second one being twice the period of the first one. The two sequences $f_{1}(n)$ and $f_{2}(n)$ are shown, for a particular $g=5 / 4$ in Fig.3.

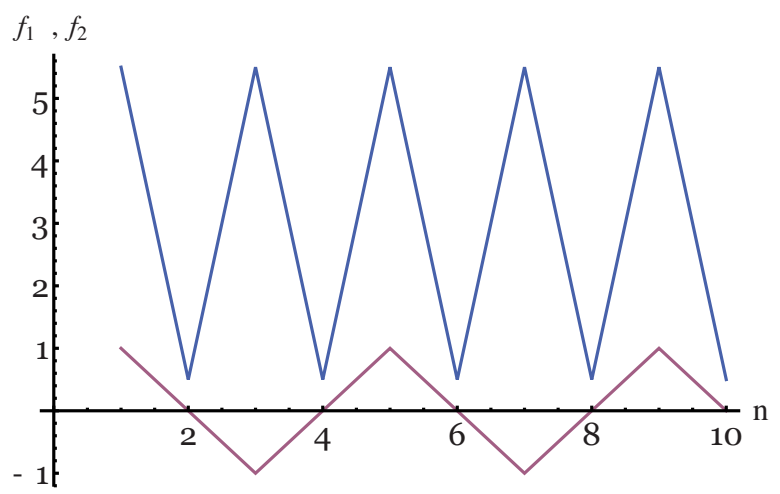

FIG. 3: Plot of $f_{1}(n)$ (upper curve) and $f_{2}(n)$ (lower curve) for the pair (b) of Eq.(50). The cumulative period is 4 .

Case (c): The solution with the period 3 . The $3^{\text {rd }}$ family of periodic solutions has period 3 and is given by:

$$
\begin{gathered}
\psi_{n}^{(1)}=r \exp \left[i\left(\frac{3}{h^{2}}+4 r^{2}\right) t\right]\left(3-2 \cos \left(\frac{2 n \pi}{3}\right)\right), \\
\psi_{n}^{(2)}=\frac{2}{h} \sqrt{\frac{9-16 h^{2} r^{2}}{5}} \exp \left[-i t \frac{\left(21+16 h^{2} r^{2}\right)}{5 h^{2}}\right] \\
\times \sin \left(\frac{2 n \pi}{3}\right),
\end{gathered}
$$

for arbitrary $r$ in the range $0<r<\frac{3}{4 h}$. These expressions can be written in an explicit form:

$$
\begin{aligned}
& \psi_{n}^{(1)}=r \exp \left[i\left(\frac{3}{h^{2}}+4 r^{2}\right) t\right] f_{1}(n), \\
& \psi_{n}^{(2)}=\sqrt{\frac{3}{5}} \sqrt{9-16 h^{2} r^{2}} \exp \left[-i t \frac{\left(21+16 h^{2} r^{2}\right)}{5 h^{2}}\right] \frac{f_{2}(n)}{h},
\end{aligned}
$$

where the sequences are

$$
f_{1}(n)=[4,4,1,4,4,1, \cdots]
$$

which is same as in the case (a), while

$$
f_{2}(n)=[1,-1,0,1,-1,0,1,-1,0, \cdots]
$$

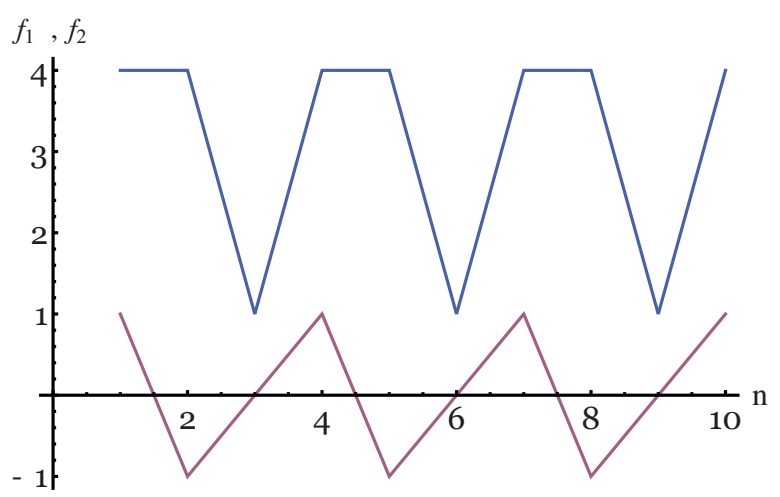

FIG. 4: Plot of the discrete functions $f_{1}$ (upper) and $f_{2}$ (lower) for the solution pair (c) of eq.(52). The period here is 3 .

which is different from the one in (a). The two latter sequences are shown in Fig.4. Symmetry relative to the exchange of variables $\psi_{n}^{(1)}(t) \leftrightarrow \psi_{n}^{(2)}(t)$ allows us to write down three more similar solutions. Clearly, in all these cases, there is no analogous coupled NLS solution, because the phase goes to $\infty$ as $h \rightarrow 0$.

\section{LOCALIZED SOLUTION}

The localized solution of the set of coupled AL equations, Eq.(45), can be written in the form:

$$
\psi_{n}^{(1)}=\frac{\sqrt{r}}{\cosh (h n p)} \exp \left(i t \frac{\left[\left(1+h^{2} r\right) \operatorname{sech}(h p)-1\right]}{h^{2}}\right),
$$

$\psi_{n}^{(2)}=\sqrt{k_{2}} \tanh (h n p) \exp \left(i k_{2} t\right)$.

where $k_{2}=h^{-2}\left[\left(1+h^{2} r\right) \operatorname{sech}^{2}(h p)-1\right]$, for arbitrary parameters $r$ and $p$.

The above solution has its analog in the form of the solution of $N=2$ Manakov (coupled NLS) set of equations. In order to see the correspondence, we take $n h=x$ and then $h \rightarrow 0$ and obtain:

$$
\begin{aligned}
& \psi^{(1)}(x, t)=\sqrt{r} \operatorname{sech}(x p) \exp \left[i t\left(r-\frac{p^{2}}{2}\right)\right], \\
& \psi^{(2)}(x, t)=\sqrt{r-p^{2}} \tanh (x p) \exp \left[i t\left(r-p^{2}\right)\right] .
\end{aligned}
$$

This effectively reproduces the solution found in [21] (see Eq.(6)).

For travelling waves, we allow non-zero velocity (i.e. $b \neq 0)$. Then the solution pair is:

$$
\begin{aligned}
& \psi_{n}^{(1)}=\sqrt{r} \operatorname{sech}(h n p-v t) \exp \left[i\left(k_{1} t+b n h\right)\right], \\
& \psi_{n}^{(2)}=\sqrt{d} \tanh (h n p-v t) \exp \left[i\left(k_{2} t+b_{2} n h\right)\right] .
\end{aligned}
$$


where

$$
\begin{aligned}
k_{1}= & \frac{1}{h^{2}}\left[-1+\left(1+h^{2} r\right) \cos (b h) \operatorname{sech}(p h)\right], \\
k_{2}= & \frac{1}{h^{2}}\left[-1+\left(1+h^{2} r\right) \operatorname{sech}^{2}(p h)\right. \\
& \quad \times \sqrt{\left.1-\operatorname{sech}^{2}(p h) \sin ^{2}(b h)\right]}, \\
v= & \frac{1}{h^{2}}\left(1+h^{2} r\right) \operatorname{sech}(p h) \sin (b h) \tanh (p h), \\
b_{2}= & \frac{1}{h} \arcsin [\operatorname{sech}(p h) \sin (b h)], \\
d= & \frac{1}{h^{2}}\left[-1+\left(1+h^{2} r\right) \operatorname{sech}^{2}(p h)\right],
\end{aligned}
$$

for arbitary parameters $b, p$ and $r$.

We take $n h=x$ and then $h \rightarrow 0$ and find that $b_{2}=b$ and $v=b p$, so the $N=2$ solution pair for the Manakov set becomes:

$\psi^{(1)}(x, t)=\sqrt{r} \operatorname{sech}[p(x-b t)] \exp \left[i\left(k_{1} t+b x\right)\right]$,

$\psi^{(2)}(x, t)=\sqrt{r-p^{2}} \tanh [p(x-b t)] \exp \left[i\left(k_{2} t+b x\right)\right]$.

where

$$
k_{1}=-\frac{1}{2}\left(b^{2}+p^{2}-2 r\right)
$$

$$
k_{2}=+r-\frac{b^{2}}{2}-p^{2}
$$

for arbitary $b, p$ and $r$.

\section{CONCLUSION}

To conclude, in this work, we have provided a direct way to establish the correspondence between the solutions of the Ablowitz-Ladik and nonlinear Schödinger equations. This technique is illustrated with a few examples and exceptions. A few pairs of important solutions are presented in Table I. In addition to the solutions considered in the text, we have also included in the table doubly-periodic solutions of the AL and NLS equations in terms of elliptic Jacobi functions. These are periodic generalizations of the heteroclinic orbits given by solutions describing modulation instability. We have also shown that the technique can be easily extended to the cases of coupled AL and NLS equations. We provide three examples of corresponding solutions of the coupled sets in Table II.

The authors acknowledge financial support of the Australian Research Council (Discovery Project No. DP110102068). N. A. is a winner of the Alexander von Humboldt Award.
[1] M. J. Ablowitz and J. F. Ladik, Stud. Appl. Math., 55, 213 (1976).

[2] M. J. Ablowitz and J. F. Ladik, Nonlinear differentialdifference equations and Fourier analysis, J. Math. Phys, 17, 1011-1018 (1976).

[3] R. Hirota, Nonlinear Partial Difference Equations. I. A difference analogue of the Korteweg - de Vries equation, Journal of the Physical Society of Japan, 43, 1424 (1977).

[4] E. Date, M. Jinbo and T. Miwa, Method for generating Discrete Soliton Equations. I, Journal of the Physical Society of Japan, 51, 4116, (1982).

[5] E. Date, M. Jinbo and T. Miwa, Method for generating Discrete Soliton Equations. II, Journal of the Physical Society of Japan, $\mathbf{5 1}, 4125$, (1982).

[6] E. Date, M. Jinbo and T. Miwa, Method for generating Discrete Soliton Equations. III, Journal of the Physical Society of Japan, 52, 388 (1983).

[7] E. Date, M. Jinbo and T. Miwa, Method for generating Discrete Soliton Equations. IV and V, Journal of the Physical Society of Japan, 52, 761 and 766 (1983).

[8] M. Hasakado, The Davey-Stewartson System and the Bäcklund transformations, Journal of the Physical Society of Japan, 67, 3038, (1998).

[9] T. Sadakane, AblowitzLadik hierarchy and twocomponent Toda lattice hierarchy, J. Phys. A: Math. Gen. 36, 8797 (2003)

[10] C. M. Schober, Phys. Lett. A, 259, 140-51 (1999).

[11] N. Akhmediev and A. Ankiewicz, Phys. Rev. E 83, 046603, (2011).
[12] A. Ankiewicz, N. Akhmediev and F. Lederer, Phys. Rev. E 83, 056602, (2011)

[13] N. Akhmediev, and A. Ankiewicz, Solitons, nonlinear pulses and beams, (Chapman \& Hall, London, 1997).

[14] M. J. Ablowitz, B. Prinari, \& A. D. Trubatch, Discrete Vector Solitons: Composite Solitons, YangBaxter Maps and Computation, Stud. in Applied Mathematics, 116, 97133 (2006).

[15] K. W. Chow, R. Conte and N. Xu, Physics Letters A, 349, 422-9 (2006).

[16] S. V. Dmitriev, P. G. Kevrekidis, N. Yoshikawa and D. J. Frantzeskakis, J. Phys. A: Math. Theor., 40, 1727-1746 (2007).

[17] A. Ankiewicz, N. Akhmediev and J. M. Soto-Crespo, Phys. Rev. E 82, 026602, (2010).

[18] N. Akhmediev, A. Ankiewicz and M. Taki, Waves that appear from nowhere and disappear without a trace, Phys. Lett. A, 373, 675-678 (2009).

[19] N. Akhmediev, A. Ankiewicz and J. M. Soto-Crespo, Rogue waves and rational solutions of the nonlinear Schrödinger equation, Phys. Rev. E 80, 026601 (2009).

[20] N. Akhmediev, V. M. Eleonskii, and N. E. Kulagin, Theoreticheskaya i Mathematicheskaya Fizika (USSR) 72, 183 (1987), English translation in: Theoretical and Mathematical Physics, 72, 809 (1987).

[21] N. Akhmediev, and A. Ankiewicz, Phys. Rev. Lett., 82, 2661-2664 (1999).

[22] A. Ankiewicz, W. Krolikowski and N. Akhmediev, Phys. Rev. E 59, 6079-6087 (1999). 
[23] N. Akhmediev and V. I. Korneev, Teoreticheskaya i Matematicheskaya Fizika (USSR), 69, 189 (1986), English translation in: [Theor. Math. Phys., 69, 1089 (1986)].

[24] K. B. Dysthe and K. Trulsen, Physica Scripta T82, 48 (1999).

[25] V. I. Shrira, V. V. Geogjaev, What makes the Peregrine soliton so special as a prototype of freak waves?. J. Eng. Math. 67, 11-22 (2010).

[26] D. H. Peregrine, Water waves, nonlinear Schrödinger equations and their solutions. J. Austral. Math. Soc. Ser. B 25, 16-43 (1983).

[27] B. Kibler, J. Fatome, C. Finot, G. Millot, F. Dias, G. Genty, N. Akhmediev, J. M. Dudley, The Peregrine soliton in nonlinear fibre optics. Nat. Phys. 6, 790-795 (2010).

[28] I. Ten and H. Tomita, Reports of RIAM Symposium
No.17SP1-2, Chikushi Campus, Kyushu University, Kasuga, Fukuoka, Japan, pp. March 10-11 (2006).

[29] N. Akhmediev, V. M. Eleonskii, and N. E. Kulagin, Zh. Eksperimentalnoii i Teoreticheskoii Fiziki, 89, 1542 (1985), english translation in: [Sov. Phys. JETP, 62, 894 (1985)].

[30] Y.C. Ma, The perturbed plane wave solutions of the cubic nonlinear Schrödinger equation. Stud. Appl. Math. 60, 43-58 (1979).

[31] N. Akhmediev, V. M. Eleonskii, N. E. Kulagin, Generation of a periodic sequence of picosecond pulses in an optical fibre: exact solutions, Zh. Eksp, i Teor. Fiz. 89, 1542-1551 (1985), English translation in: [Sov. Phys. JETP, 61, 894-899 (1985)].

[32] E. A. Kuznetsov, Dokl. Akad. Nauk SSSR, pp. 575-577 (1977). 
TABLE I: Comparison of selected exact solutions of the NLS and the A-L equations

Appendix 1: Corresponding sets of exact solutions of a single equation

Nonlinear Schrödinger equation (NLS):

$$
i \frac{\partial \psi}{\partial t}+\frac{1}{2} \psi_{x x}+|\psi|^{2} \psi=0
$$

Ablowitz - Ladik equation with a free parameter $h$ :

$$
\begin{aligned}
i \frac{\partial \psi_{n}^{(1)}}{\partial t}+\frac{1}{2 h^{2}}\left(\psi_{n-1}^{(1)}\right. & \left.+\psi_{n+1}^{(1)}-2 \psi_{n}^{(1)}\right) \\
& +\frac{1}{2}\left(\psi_{n-1}^{(1)}+\psi_{n+1}^{(1)}\right)\left|\psi_{n}^{(1)}\right|^{2}=0
\end{aligned}
$$

One soliton soltion of NLS:

$\psi(x, t)=p \operatorname{sech}(x p) \exp \left(i p^{2} \frac{t}{2}\right)$
One soliton solution of AL equation:

$\psi_{n}(t)=\frac{\sinh (h p)}{h} \operatorname{sech}(h n p) \exp \left[\frac{2 i t}{h^{2}} \sinh ^{2}\left(\frac{h p}{2}\right)\right]$.

Growth-return cycle of modulation instability for NLS (Akhmediev breather):

$$
\psi(x, t)=q\left[\kappa \frac{\kappa^{2} \cosh \left(\delta_{1} q^{2} t\right)+2 i \delta_{1} \sinh \left(\delta_{1} q^{2} t\right)}{2 \kappa \cosh \left(\delta_{1} q^{2} t\right) \pm 2 \delta_{1} \cos (\kappa q x)}-1\right] e^{i q^{2} t}
$$$$
\frac{1}{h}\left[\frac{2\left(1+h^{2} q^{2}\right) w \cosh \left(\frac{\delta t}{2 h^{2}}\right)+i \frac{\delta}{2} \sinh \left(\frac{\delta t}{2 h^{2}}\right)}{q h \cosh \left(\frac{\delta t}{2 h^{2}}\right) \pm c_{1} \cos (\kappa q n h)}-q h\right] e^{i q^{2} t}
$$

Growth-return cycle of modulation instability with maximum growth rate [23]:

$\psi(x, t)=q \frac{\cos (\sqrt{2} q x)+i \sqrt{2} \sinh \left(q^{2} t\right)}{\sqrt{2} \cosh \left(q^{2} t\right)-\cos (\sqrt{2} q x)} e^{i q^{2} t}$
Modulation instability with maximum growth rate [12]; eq.(26):
First order rational solution [26]:

$\psi(x, t)=q\left(\frac{4\left(1+2 i q^{2} t\right)}{1+4 q^{2} x^{2}+4 q^{4} t^{2}}-1\right) e^{i q^{2} t}$

Soliton on a background [30]:

$\psi(x, t)=e^{i q^{2} t}\left[k_{2} \frac{k_{2} \cos \left(\frac{k_{2}}{2} d t\right)+i d \sin \left(\frac{k_{2}}{2} d t\right)}{ \pm d \cosh \left(k_{2} x\right)-2 q \cos \left(\frac{k_{2}}{2} d t\right)}-q\right]$

Doubly-periodic breather of NLS equation [20, 23]:

$\psi(x, t)=\frac{r}{\sqrt{2}} \exp \left(i k_{1} \frac{r^{2}}{2} t\right) \times$

$$
\frac{\left(\frac{1-k_{1}}{2}\right)^{1 / 4} \operatorname{dn}\left(\frac{r^{2}}{2} t, m_{1}\right) \operatorname{cn}\left(r x, \frac{1-k_{1}}{2}\right)+i \sqrt{k_{1}} k_{t} \operatorname{sn}\left(\frac{r^{2}}{2} t, m_{1}\right)}{k_{t}-\left(\frac{1-k_{1}}{2}\right)^{1 / 4} \sqrt{k_{1}} \operatorname{cn}\left(\frac{r^{2}}{2} t, m_{1}\right) \operatorname{cn}\left(r x, \frac{1-k_{1}}{2}\right)}
$$

where $r$ and $k_{1}$ are independent parameters; $m_{1}=k_{1}^{2}$.

Periodic stationary solutions of NLS equation :

$\psi(x, t)=\sqrt{a} \operatorname{dn}(b x, m) \exp (i k t), E q .(19)$

$\psi(x, t)=0$

$\psi(x, t)=\sqrt{a} \operatorname{cn}(b x, m) \exp (i k t), E q \cdot(25)$ $\psi_{n}(t)=q \frac{\sqrt{1+h^{2} q^{2}} \cos (v n h)+i \sqrt{2+h^{2} q^{2}} \sinh \left(q^{2} t\right)}{\sqrt{2+h^{2} q^{2}} \cosh \left(q^{2} t\right)-\sqrt{1+h^{2} q^{2}} \cos (v n h)} e^{i q^{2} t}$

First order rational solution [11], [12] :

$\psi_{n}^{(1)}(t)=q\left(\frac{4\left(1+h^{2} q^{2}\right)\left(1+2 i q^{2} t\right)}{1+4 h^{2} q^{2}\left(n^{2}+q^{4} t^{2}\right)+4 q^{4} t^{2}}-1\right) e^{i q^{2} t}$

Soliton on a background [12]; eq.(31) :

$\psi_{n}(t)=\frac{1}{h} e^{i q^{2} t}\left[\frac{\cos \left(\frac{w_{1} t}{2 h^{2}}\right)+i s_{6} \sin \left(\frac{w_{1} t}{2 h^{2}}\right)}{s_{3}\left[-\cos \left(\frac{w_{1} t}{2 h^{2}}\right) \pm s_{5} \cosh \left(k_{2} n h\right)\right]}-q h\right]$.

Doubly-periodic breather of AL equation [12]:

$\psi_{n}(t)=q \exp \left(i k_{1} q^{2} t\right) \times$

$\frac{\sqrt{k} \operatorname{dn}\left(q^{2} t, m_{1}\right) \operatorname{cn}(r n h, m)+i \sqrt{k_{1}} k_{t} \operatorname{sn}\left(q^{2} t, m_{1}\right)}{k_{t}-\sqrt{k k_{1}} \operatorname{cn}\left(q^{2} t, m_{1}\right) \operatorname{cn}(r n h, m)}$, $k_{t}-\sqrt{k k_{1}} \mathrm{cn}\left(q^{2} t, m_{1}\right) \mathrm{cn}(r n h, m)$

"Periodic" stationary solutions of AL equation : $\psi_{n}^{(t)}=\sqrt{a} \operatorname{dn}(b n h, m) \exp (i k t)$, Eqs.(16) - (18)

$\psi_{n}^{(t)}=\sqrt{a} \operatorname{sn}(b n h, m) \exp (i k t), E q \cdot(20)$

$\psi_{n}^{(t)}=\sqrt{a} \operatorname{cn}(b n h, m) \exp (i k t), E q s .(22)-(24)$
There is no continuous analog

There is no continuous analog

There is no continuous analog
Strictly periodic solutions of AL equation:

$\psi_{n}^{(1)}=\psi_{n}^{(1)}=c \sin \left(\frac{n \pi}{2}\right) \exp \left(-i t / h^{2}\right)$,

$\psi_{n}^{(1)}=c \sin \left(\frac{n \pi}{3}\right) \exp \left[i\left(\frac{3 c^{2}}{8}-\frac{1}{2 h^{2}}\right) t\right]$,

$\psi_{n}^{(1)}=c \sin \left(\frac{2 n \pi}{3}\right) \exp \left[-\frac{3 i}{8}\left(c^{2}+\frac{4}{h^{2}}\right) t\right]$, 
TABLE II: Comparison of exact solutions of the coupled set of NLS and the coupled set of A-L equations

\begin{tabular}{|c|c|}
\hline \multicolumn{2}{|c|}{ Appendix 2: Corresponding sets of exact solutions of a set of equations } \\
\hline $\begin{array}{l}\text { Coupled set of nonlinear Schrödinger equations } \\
\text { (see [20],[21],[22]) } \quad(j=1,2) \text { : } \\
i \frac{\partial \psi_{j}}{\partial t}+\frac{1}{2} \frac{\partial^{2} \psi_{j}}{\partial x^{2}}+\psi_{j}\left(\sum_{m=1}^{N}\left|\psi_{m}\right|^{2}\right)=0\end{array}$ & $\begin{array}{l}\text { Coupled Ablowitz-Ladik set of equations } \\
\text { (present work) Eqs.(45), } \quad(j=1,2) \text { : } \\
\begin{aligned} i \frac{\partial \psi_{n}^{(j)}}{\partial t}+ & \frac{1}{2 h^{2}}\left(\psi_{n-1}^{(j)}+\psi_{n+1}^{(j)}-2 \psi_{n}^{(j)}\right) \\
& +\frac{1}{2}\left(\psi_{n-1}^{(j)}+\psi_{n+1}^{(j)}\right)\left(\sum_{m=1}^{N}\left|\psi_{n}^{(m)}\right|^{2}\right)=0\end{aligned}\end{array}$ \\
\hline $\begin{array}{l}\text { Manakov pair solution [21]; Eqs.(54): } \\
\psi^{(1)}(x, t)=\sqrt{r} \operatorname{sech}(x p) \exp \left[i t\left(r-\frac{p^{2}}{2}\right)\right] \\
\psi^{(2)}(x, t)=\sqrt{r-p^{2}} \tanh (x p) \exp \left[i t\left(r-p^{2}\right)\right] .\end{array}$ & $\begin{array}{l}\text { Discrete pair solution - Eqs. (53) of this work: } \\
\psi_{n}^{(1)}=\sqrt{r} \operatorname{sech}(h n p) \exp \left(\frac{i t}{h^{2}}\left[\left(1+h^{2} r\right) \operatorname{sech}(h p)-1\right]\right), \\
\psi_{n}^{(2)}=\sqrt{k_{2}} \tanh (h n p) \exp \left(i t k_{2}\right) .\end{array}$ \\
\hline $\begin{array}{l}\text { travelling Manakov pair solution, Eqs.(56): } \\
\psi^{(1)}(x, t)=\sqrt{r} \operatorname{sech}[p(x-b t)] \exp \left[i\left(k_{1} t+b x\right)\right], \\
\psi^{(2)}(x, t)=\sqrt{r-p^{2}} \tanh [p(x-b t)] \exp \left[i\left(k_{2} t+b x\right)\right] .\end{array}$ & $\begin{array}{l}\text { moving discrete pair solution - Eqs.(55) of this work: } \\
\psi_{n}^{(1)}=\sqrt{r} \operatorname{sech}(h n p-v t) \exp \left[i\left(k_{1} t+b n h\right)\right] \\
\psi_{n}^{(2)}=\sqrt{d} \tanh (h n p-v t) \exp \left[i\left(k_{2} t+b_{2} n h\right)\right]\end{array}$ \\
\hline
\end{tabular}

